OPEN ACCESS

Edited by:

Gurvinder Kaur,

All India Institute of Medical

Sciences, India

Reviewed by:

Edward Seth Kraus, Johns Hopkins University,

United States

Gaurav Gupta,

Virginia Commonwealth University,

United States

*Correspondence:

Yong Ren

koalary@qq.com

Ning Na

naing@mail.sysu.edu.cn

tThese authors have contributed equally to this work

Specialty section:

This article was submitted to

Nephrology,

a section of the journal

Frontiers in Medicine

Received: 05 March 2021 Accepted: 06 December 2021 Published: 18 January 2022

Citation:

Luo Y, Liang J, Hu X, Tang Z, Zhang J,

Han L, Dong Z, Deng W, Miao B, Ren $Y$ and Na N (2022) Deep Learning

Algorithms for the Prediction of

Posttransplant Renal Function in Deceased-Donor Kidney Recipients: A

Preliminary Study Based on Pretransplant Biopsy.

Front. Med. 8:676461.

doi: 10.3389/fmed.2021.676461

\section{Deep Learning Algorithms for the Prediction of Posttransplant Renal Function in Deceased-Donor Kidney Recipients: A Preliminary Study Based on Pretransplant Biopsy}

You Luo ${ }^{1 \dagger}$, Jing Liang ${ }^{2+}$, Xiao Hu ${ }^{1+}$, Zuofu Tang ${ }^{1}$, Jinhua Zhang ${ }^{1}$, Lanqing Han ${ }^{3}$, Zhanwen Dong ${ }^{1}$, Weiming Deng ${ }^{1}$, Bin Miao ${ }^{1}$, Yong Ren ${ }^{3,4 *}$ and $\mathrm{Ning} \mathrm{Na}^{1 *}$

\footnotetext{
1 Department of Kidney Transplantation, The Third Affiliated Hospital of Sun Yat-sen University, Guangzhou, China, ${ }^{2}$ Department of Pathology, The Third Affiliated Hospital of Sun Yat-sen University, Guangzhou, China, ${ }^{3}$ Artificial Intelligence Innovation Center, Research Institute of Tsinghua, Pearl River Delta, Guangzhou, China, ${ }^{4}$ Guangdong Provincial Key Laboratory of Digestive Cancer Research, The Seventh Affiliated Hospital of Sun Yat-sen University, Shenzhen, China
}

Background: Posttransplant renal function is critically important for kidney transplant recipients. Accurate prediction of graft function would greatly help in deciding acceptance or discard of allocated kidneys.

Methods : Whole-slide images (WSIs) of H\&E-stained donor kidney biopsies at $\times 200$ magnification between January 2015 and December 2019 were collected. The clinical characteristics of each donor and corresponding recipient were retrieved. Graft function was indexed with a stable estimated glomerular filtration rate (eGFR) and reduced graft function (RGF). We used convolutional neural network (CNN)-based models, such as EfficientNet-B5, Inception-V3, and VGG19 for the prediction of these two outcomes.

Results: In total, 219 recipients with H\&E-stained slides of the donor kidneys were included for analysis [biopsies from standard criteria donor (SCD)/expanded criteria donor (ECD) was 191/28]. The results showed distinct improvements in the prediction performance of the deep learning algorithm plus the clinical characteristics model. The EfficientNet-B5 plus clinical data model showed the lowest mean absolute error (MAE) and root mean square error (RMSE). Compared with the clinical data model, the area under the receiver operating characteristic (ROC) curve (AUC) of the clinical data plus image model for eGFR classification increased from 0.69 to 0.83. In addition, the predictive performance for RGF increased from 0.66 to 0.80 . Gradient-weighted class activation mappings (Grad-CAMs) showed that the models localized the areas of the tubules and interstitium near the glomeruli, which were discriminative features for RGF.

Conclusion: Our results preliminarily show that deep learning for formalin-fixed paraffin-embedded H\&E-stained WSIs improves graft function prediction accuracy for deceased-donor kidney transplant recipients.

Keywords: kidney transplantation, deceased donor, graft function, deep learning, whole slide digital image 


\section{INTRODUCTION}

Kidney transplantation remains the best option for patients with end-stage renal disease (ESRD). However, due to a shortage of organs, the use of marginal kidneys is rising (1), and as a result, the incidence of complications is gradually increasing. Graft function (estimated glomerular filtration rate [eGFR]) is one of the most crucial risk factors for surgical complications and longterm survival. It has been indicated that kidney function after transplantation correlates strongly with graft long-term survival (2-4). Graft function is the core cornerstone linked to long-term survival and plays a crucial role in assessing transplant results $(5,6)$. Posttransplantation graft function is determined by the donor, procurement and preservation, recipient characteristics, and postsurgical treatment, though there is currently no stable model for predicting allograft functional outcomes. Pretransplant graft discard is influenced by the experience of transplant surgeons based on donor characteristics, with or without biopsy evaluation, and assessment of the donor kidneys mainly involves clinical scores and histological evaluation. In general, clinical score evaluation began with standard criteria donor (SCD)/expanded criteria donor (ECD) binary classification and has evolved to the currently widely used kidney donor risk index (KDRI). The discriminative power of such a clinical score is low to moderate, and the use of KDRI in the United States leads to a high discard rate of donated kidneys. Indeed, in our clinical practice, low-KDRI kidneys do not always regenerate satisfactory graft function, whereas many high-KDRI kidneys regenerate very good renal function. Overall, the predictive performance of using net clinical features for graft function outcome is relatively poor (7), though the addition of histopathological features may improve the predictive performance of graft outcome. Pretransplant histological evaluation of the donor kidneys is critically important for kidney quality evaluation, especially for marginal kidneys. The histological score composites include the glomerulus, tubule, interstitium, and vessels (mainly artery). Nonetheless, the usefulness of such a score remains controversial due to its low predictive power for transplant outcomes $(8,9)$.

Pretransplant biopsy by H\&E staining is crudely evaluated for acute kidney injury and glomerular sclerosis, depending on the experience of the pathologist, and is variable. Pathological evaluations of the donor kidneys from different pathologists/nephrologists and from different levels of pathologists disagree. For example, a previous study showed good reproducibility regarding glomerulus number and sclerosis percentage but very poor or fair intraclass correlation regarding interstitial fibrosis, tubular atrophy, interstitial inflammation, arteriolar thrombi, and arterial intimal fibrosis, especially for slides from needle and frozen biopsies. The Banff Working Group suggests that training of general pathologists to assess

\footnotetext{
Abbreviations: WSIs, Whole-slide images; CIE, clinical information extraction; $\mathrm{H} \& \mathrm{E}$, hematoxylin-eosin; PAS, periodic acid-Schiff; eGFR, estimated glomerular filtration rate; $\mathrm{CNN}$, convolutional neural network; MAE, mean absolute error; RMSE, root mean square error; ROC, receiver operating characteristic; DGF, delayed graft function; SGF, slow graft function; AI, artificial intelligence; COTRS, China Organ Transplant Response System; ROIs, regions of interest; ECD, expanded criteria donor; KDRI, Kidney Donor Risk Index.
}

donor biopsies using consistent criteria should be pursued, and adoption of rapid formalin-fixation and paraffin-embedding protocols may potentially reduce interference for frozen slides (10). These recommendations might increase the reliability of histopathological evaluation, and an automatic analytic machine with deep learning algorithms may assume this role in analyzing the donor biopsies.

The use of artificial intelligence (AI) is increasing explosively in the medical field. Deep learning methods, such as convolutional neural networks (CNNs), are very practical for image and audio analysis (11). Inspired by these ideas, we aimed to explore whether the combination of kidney biopsy whole-slide images (WSIs) and clinical features can provide more information for transplant outcome prediction.

\section{MATERIALS AND METHODS}

\section{Study Population and Clinical Variable Data}

Deceased-donor kidney recipients at the Third Affiliated Hospital of Sun Yat-sen University, Guangzhou, China from January 2015 to December 2019 were retrospectively reviewed. In total, 243 donor kidney recipients underwent pretransplantation biopsy. Demographic data for the recipients and donors were collected from China Organ Transplant Response System (COTRS) and the hospital information system (HIS). COTRS is the sole legitimate official registry platform designated by the National Health Commission of China for solid organ donation from deceased-citizen donors, matching, and allocation. After screening, 219 recipients were included in the final analysis. The screening process is depicted in Figure 1. This study was approved by the research ethics committee (IRB No. [2020]02243-01) and was compliant with the Declaration of Helsinki. The clinical and research activities being reported are consistent with the Principles of the Declaration of Istanbul as outlined in the "Declaration of Istanbul on Organ Trafficking and Transplant Tourism."

Delayed graft function (DGF) was defined as requiring dialysis within the first week after transplantation (12). Slow graft function (SGF) was defined as serum creatinine at postoperative day 7 (POD7) $\geq 2.5 \mathrm{mg} / \mathrm{dl}$, and immediate graft function (IGF) was defined as creatinine at POD7 $<2.5 \mathrm{mg} / \mathrm{dl}$ (13). Reduced graft function (RGF) was a composite endpoint consisting of DGF and SGF. The renal function of each recipient was indexed with the eGFR and comprehensively evaluated based on serum creatinine (or calculated eGFR) based on each followup monitoring visit within the first year. In this study, we used stable eGFR as the graft outcome. The eGFR of recipient was calculated by the Chronic Kidney Disease-Epidemiology (CKDEPI) algorithm (14). Stable eGFR was defined as the approximate median value where the monitoring results fluctuated within the first year (3 12 months). Stable eGFR was evaluated from the best period that the graft function of recipient was reached by two senior physicians and not the whole year of monitoring results. Recipients who died during the perioperative period, accepted donations from other organ procurement organizations (OPOs), or were lost to follow-up were excluded. The case of a recipient with a primary non-functional kidney was labeled 

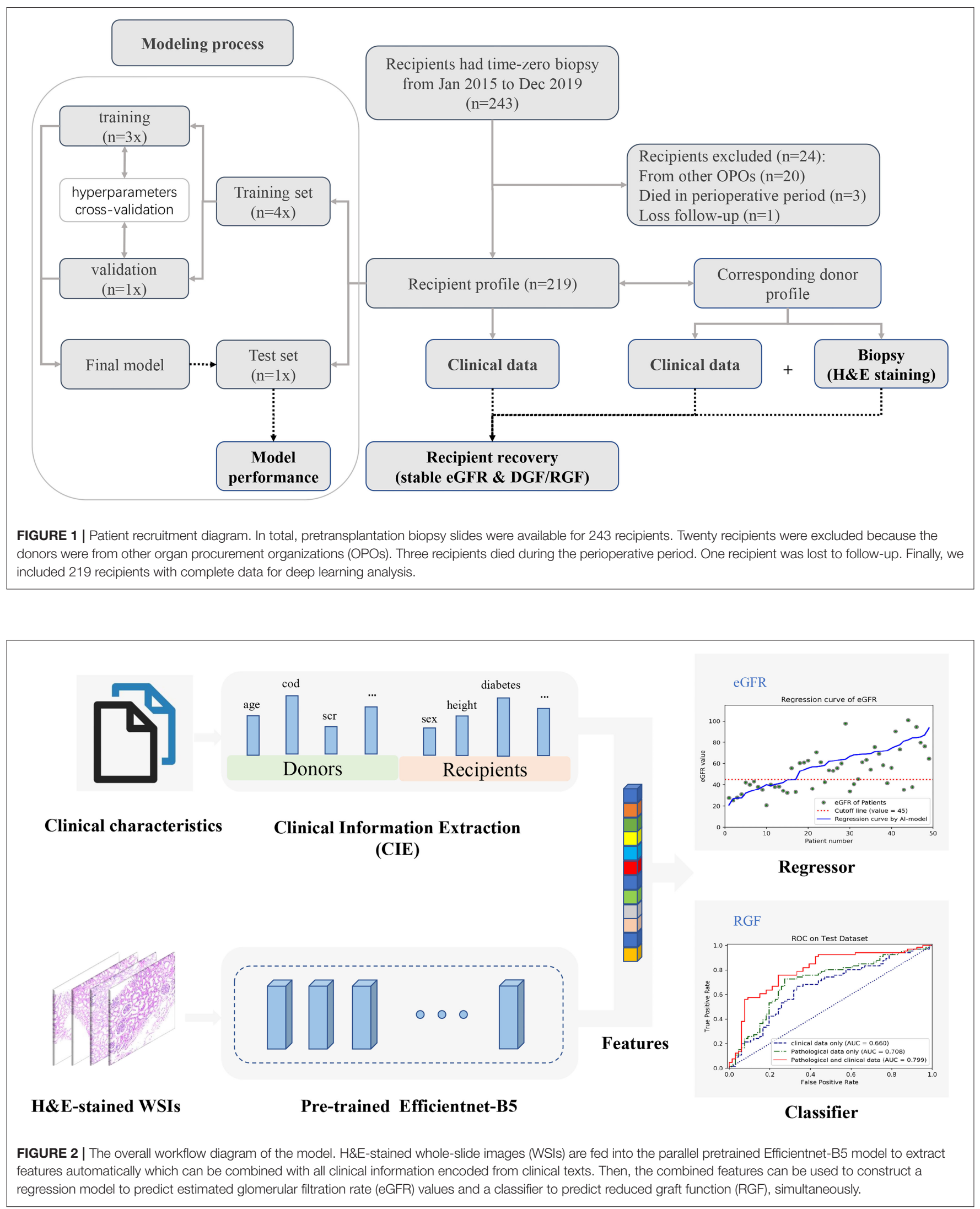


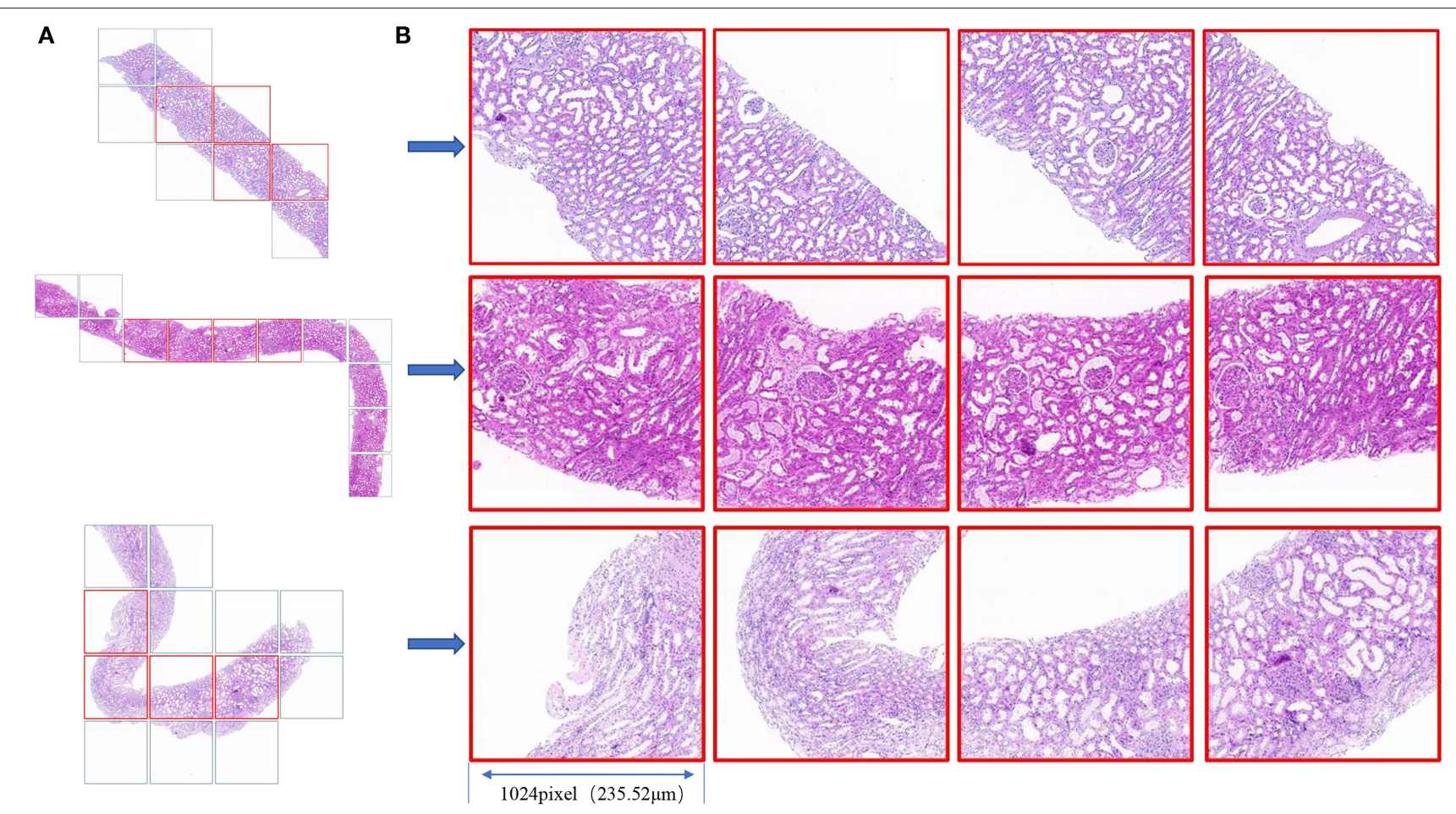

FIGURE 3 | (A) Biopsy tissue was scanned as a WSI under a $\times 20$ objective lens, and the slide window with a 1,024 window width was used for tiling the WSI to patches (square box). (B) The four patches with the largest proportion of pathological tissue in each WSI are framed in red. The field of view of each square patch is $1,024 \times 1,024$ pixels, corresponding to $235.52 \times 235.52 \mu \mathrm{m}^{2}$.

with the last eGFR before returning to dialysis. Additionally, we arbitrarily divided stable graft function into binary categories by a cutoff of $45 \mathrm{ml} / \mathrm{min} / 1.73 \mathrm{~m}^{2}$ as sensitivity analysis. Based on previous publications, the graft failure hazard rises sharply over this point $(2-4,7,15)$. The clinical characteristics screened for the predictive model building included both donor and recipient features. These features included recipient age, sex, height, weight, transplant history, primary cause of ESRD, diabetes, dialysis modality, dialysis vintage, positive panel reaction antibody (PRA), human leukocyte antigen (HLA) mismatch level, and donor characteristics of cold ischemia hours, warm ischemia minutes, cause of death, death type, donated kidney, donor sex, age, height, weight, terminal serum creatinine level, and KDRI. Missing values in the HLA mismatch level were deemed one category.

\section{Biopsy Samples and Model Training Pathological WSIs}

We reviewed all H\&E-stained (formalin-fixed paraffinembedded: FFPE) pretransplantation biopsy slides and scanned and stored the WSIs at $\times 200$ magnification with a resolution of $0.23 \mu \mathrm{m} /$ pixel using an automatic digital slide scanner (Panoramic 250 FLASH, 3DHISTECH Ltd, Budapest, Hungary). In total, 219 recipients with $\mathrm{H} \& \mathrm{E}$-stained slides of the donor kidneys were included in the final analysis. All biopsies were procedurally performed using the 18-gauge needles during donor kidney trimming (i.e., all samples were preimplantation biopsies). Pathological scores (Remuzzi scores) were evaluated by a single pathologist (Jing Liang).

\section{Deep Learning and Transfer Learning}

A CNN-based model was used to extract the features of the H\&E WSIs, such that important information could be obtained for regression fitting. EfficientNets is a new baseline network designed by neural architecture search and consists of a family of models. From B0 to B7, the corresponding accuracy increases, but the number of parameters also increases, which leads to a decrease in training and deployment efficiency. The EfficientNet B5 model was considered to be the most suitable base model due to the trade-off between few parameters and sufficiently high ImageNet Top-1 accuracy (Top-1 accuracy means that the model with the highest probability of prediction must be exactly the true classification). Correspondingly, Top-5 accuracy indicates that any part of the model that yields the 5 highest probability predictions should include the true classification. To find the most suitable model for predicting stable eGFR and RGF, the performances of EfficientNet-B5, Inception-V3 and VGG19 were compared, and EfficientNet-B5 was selected as the base network due to its superior performance. Regions of interests (ROIs) patches selected from H\&E-stained renal biopsy tissues were first sent into EfficientNet-B5 to extract high-dimensional pathological image features, and the corresponding clinical texts of patient preprocessed by one-hot coding conversion and normalization were then synchronously input into a fully 
connected network (FCN) to extract the clinical information. The whole process is illustrated in Figure 2.

To perform feature fusion, the extracted clinical information (CIE), i.e., clinical data, was spliced with the penultimate layer of the EfficientNet-B5 network; next, a new layer containing only one neuron was added to regress the eGFR values, and a parallel layer containing two neurons was added to classify the RGF state. It should be noted that for regression tasks, the neurons in the last layer do not use any activation function such that the model can output unlimited prediction values. This model, which includes clinical information and a $\mathrm{CNN}$, is called the EfficientNet-B5 + CIE model, where the CIE model denotes the clinical information extraction model. After obtaining the regression model, we classified the actual value and predicted value according to cutoff values (eGFR of 45 $\mathrm{ml} / \mathrm{min} / 1.73 \mathrm{~m}^{2}$ ). With the help of the RGF classification model and the indirect eGFR classification model, postoperative recovery can be qualitatively evaluated. In addition, the sample size of medical pathological images is relatively small compared with the ImageNet dataset (http://www.image-net. org/). To avoid overfitting, we used transfer learning to initialize all parameters of the $\mathrm{CNN}$ model with ImageNet trained parameters before training and then updated the parameters via backpropagation during training. In conclusion, we used both clinical data and histopathological images to build neural network models to predict stable eGFR and RGF. We preset to test six prediction models, such as a model based on clinical data (CIE model), a model based on clinical data and the histopathological Remuzzi score (CIE+PRS model), models based on histopathological images (VGG19 model, InceptionV3 model, and EfficientNet-B5 model), and a model based on clinical data and histopathological images (EfficientNetB5+CIE model).

\section{Implementation Details}

We used Python 3.6 as the programming language and Keras 2.2 as the deep learning architecture. We trained and tested our models using one Nvidia Tesla V100 GPU with 32 GB memory with the help of NumPy, Matplotlib, and scikit-learn. Cases with each H\&E-stained WSI were randomly divided into a training dataset, validation dataset, and independent test dataset at a ratio of $3: 1: 1$. First, we used the validation dataset to choose hyperparameters, such as the number of epochs and the learning rate. After the parameters were determined, the validation data and training data were combined to form a new training dataset for retraining the model, and the performance of the model was evaluated using the independent test dataset (as shown in Figure 1). Four patches with the largest proportion of pathological tissue in each H\&E-stained WSI were selected as ROIs through OpenSlide 1.9 using a sliding window with window width set as 1,024 pixels (as shown in Figure 3). A total of 876 patches were obtained. Before inputting the models, the resolution was adjusted to 256 pixels and normalized. Our models adopted the Adam optimizer with a learning rate of $8 \mathrm{e}-4$. The best model was saved when the mean absolute error (MAE) for the test dataset was the lowest in 500 epochs.

\section{Model Performance Evaluation Metrics}

For regression prediction tasks, the MAE, root mean square error (RMSE), R-squared value, and explained variance score (EVS) were calculated. The MAE is the mean value of the absolute value of error between the real value and the predicted value. The RMSE is the square of the difference between the real value and the predicted value; the sum is averaged, and the square root is obtained. The R-squared value and EVS represent the degree of fitting, and the values range from 0 to 1 . The higher the value is, the better the fit is.

For classification prediction tasks, the confusion matrix and receiver operating characteristic (ROC) curve were generated. The confusion matrix is used to visually evaluate the performance of deep learning algorithms. The sensitivity, specificity, positive predictive value, and negative predictive value can be calculated by means of the confusion matrix. The ROC curve is depicted by plotting the true positive rate (TPR, sensitivity) vs. the falsepositive rate (FPR, 1-specificity) at various threshold settings. Accuracy is assessed by the area under the ROC curve (AUC).

\section{Statistical Analysis}

The demographics of the donors and recipients are presented as frequencies for categorical variables and medians (interquartile ranges [IQRs]) for continuous variables. Differences were explored using the Wilcoxon rank-sum tests (skewed distribution) or the $t$-test (normal distribution) for continuous variables and Fisher's exact tests for categorical variables. The statistical analyses were performed using R 4.0.2 (R Foundation for Statistical Computing, Vienna, Austria).

\section{RESULTS}

\section{Population Characteristics}

In total, 219 recipients underwent donor kidney biopsy, and complete follow-up was included in the analysis. All recipients were prescribed traditional triple immunosuppression regimens (CNI+ MPA+ glucocorticoids). The features used for the predictive model building are shown in Table 1. In this population, the incidence of DGF and RGF was 25/219 (11.4\%) and $92 / 219(42 \%)$, respectively. Due to the large imbalance in DGF proportion, we did not set DGF as an analytical target for deep learning and used RGF instead. The median stable eGFR was $61.2[47.1,76.9] \mathrm{ml} / \mathrm{min} / 1.73 \mathrm{~m}^{2}$ during followup visits. Donor death category, donor cause of death KDRI, terminal creatinine, warm ischemia minutes, cold ischemia hours, recipient sex, weight, Remuzzi scores, and stable eGFR were significantly different between the RGF groups. Compared with RGF recipients, the IGF group had better stable eGFR, lower body weight, a higher proportion of female recipients, lower warm ischemia minutes and cold ischemia hours, lower donor KDRI and terminal creatinine levels, and a higher proportion of trauma donors and DBD donors. These results were consistent with previous results. Figure 4 displays the serum creatinine level (Figure 4A) and eGFR level (Figure 4B) of two recipient groups at each time point during the first year follow-up. The trend curve shows better graft function recovery in IGF recipients than in RGF recipients. 
TABLE 1 | Distributions of the patient baseline characteristics.

\begin{tabular}{|c|c|c|c|c|}
\hline Characters & IGF $(N=127)$ & RGF $(N=92)$ & Total $(N=219)$ & $P$-value \\
\hline Stable eGFR & $68.8(55.4,82.4)$ & $47.4(38.0,62.1)$ & $61.2(47.1,76.9)$ & $<0.01$ \\
\hline DGF & & & & $<0.01$ \\
\hline No & 127 & 67 & 194 & \\
\hline \multicolumn{5}{|l|}{ Donor } \\
\hline Female & 25 & 21 & 46 & \\
\hline Male & 102 & 71 & 173 & \\
\hline Weight (kg) & $60(55,70)$ & $65(55,75)$ & $64(55,71)$ & 0.31 \\
\hline Height (cm) & $167(162,172)$ & $168(160,170)$ & $168(161,172)$ & 0.75 \\
\hline Donor death & & & & 0.006 \\
\hline SCD & 112 & 79 & 191 & \\
\hline ECD & 15 & 13 & 28 & \\
\hline Cause of death & & & & $<0.01$ \\
\hline CVD & 50 & 59 & 109 & \\
\hline Trauma & 70 & 27 & 97 & \\
\hline Other & 7 & 6 & 13 & \\
\hline KDRI & $1.31(1.09,1.53)$ & $1.50(1.34,1.71)$ & $1.40(1.21,1.62)$ & $<0.01$ \\
\hline Terminal creatinine ( $\mu \mathrm{mol} / \mathrm{L})$ & $103(70,165)$ & $187(103,301)$ & $120(76.5,200)$ & $<0.01$ \\
\hline Kidney & & & & 0.49 \\
\hline Left & 61 & 49 & 110 & \\
\hline Right & 66 & 43 & 109 & \\
\hline PRA & & & & 0.53 \\
\hline Positive & 13 & 12 & 25 & \\
\hline Negative & 114 & 80 & 194 & \\
\hline CIT (h) & $7.7(6.7,9.5)$ & $8.9(7.3,10.9)$ & $8.2(6.9,10.2)$ & 0.01 \\
\hline WIT (min) & $6(0,10)$ & $10(0,11)$ & $7(0,10)$ & 0.02 \\
\hline \multicolumn{5}{|l|}{ Recipient } \\
\hline Age & $40(34,49)$ & $41.5(33,48)$ & $40(33.5,48.5)$ & 0.88 \\
\hline Sex & & & & 0.01 \\
\hline Female & 45 & 17 & 62 & \\
\hline Male & 82 & 75 & 157 & \\
\hline Weight (kg) & $58(52,67.3)$ & $60(54.5,70.3)$ & $60(53.5,69.8)$ & 0.05 \\
\hline Height (cm) & $168(162,170)$ & $168(165,171)$ & $168(163,170)$ & 0.07 \\
\hline Transplantation history & & & & 0.24 \\
\hline Yes & 2 & 4 & 6 & \\
\hline No & 125 & 88 & 213 & \\
\hline Cause of ESRD & & & & 0.32 \\
\hline Glomerulonephritis & 107 & 74 & 181 & \\
\hline DN & 6 & 10 & 16 & \\
\hline
\end{tabular}


TABLE 1 | Continued

\begin{tabular}{|c|c|c|c|c|}
\hline Characters & IGF $(N=127)$ & RGF $(N=92)$ & Total $(N=219)$ & $P$-value \\
\hline HTN & 5 & 4 & 9 & \\
\hline Others & 9 & 4 & 13 & \\
\hline Diabetes & & & & 0.68 \\
\hline Yes & 15 & 13 & 28 & \\
\hline No & 112 & 79 & 191 & \\
\hline PD & 28 & 16 & 44 & \\
\hline No dialysis & 16 & 5 & 21 & \\
\hline Dialysis vintage & & & & 0.33 \\
\hline No dialysis & 16 & 5 & 21 & \\
\hline $1 \sim 6$ months & 34 & 24 & 58 & \\
\hline Tac & 110 & 69 & 179 & \\
\hline CsA & 17 & 23 & 40 & \\
\hline MPA & 127 & 92 & 219 & 1.00 \\
\hline Glucocorticoids & 127 & 92 & 219 & 1.00 \\
\hline \multicolumn{5}{|l|}{ Pathology (Remuzzi score) } \\
\hline Glomerular global sclerosis & & & & $<0.01$ \\
\hline 0 & 67 & 41 & 108 & \\
\hline 1 & 50 & 27 & 77 & \\
\hline 2 & 10 & 20 & 30 & \\
\hline 3 & 0 & 4 & 4 & \\
\hline Tubular atrophy & & & & $<0.01$ \\
\hline 2 & 0 & 1 & 1 & \\
\hline 3 & 0 & 0 & 0 & \\
\hline Arterial and arteriolar narrowing & & & & 0.01 \\
\hline 0 & 96 & 52 & 148 & \\
\hline 1 & 27 & 29 & 56 & \\
\hline 2 & 4 & 10 & 14 & \\
\hline 3 & 0 & 1 & 1 & \\
\hline
\end{tabular}

DGF, delayed graft function; DBD, donation after brain death; DCD, donation after circulatory death; CVD, cerebrovascular disease; CNS, central nervous system; KDRI, Kidney Donor

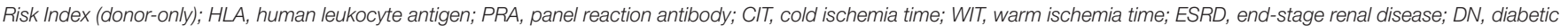

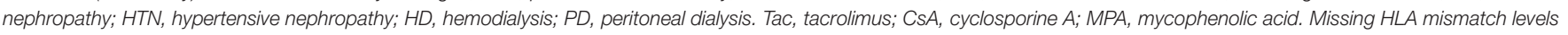
usually occurred at emergent procurement, and donor HLA results were not registered in COTRS.

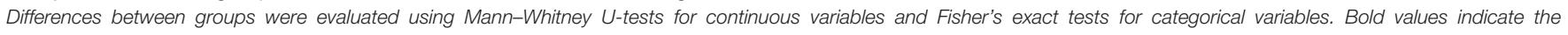
statistical significance.

\section{Prediction of Graft Function Recovery}

Regression methods were employed to predict the stable eGFR, and the concordance between the predicted value and true label and the predictive accuracy are listed in Table 2. The EfficientNet-B5+CIE model showed the best predictive accordance and accuracy; it had the lowest MAE (12.52) and RMSE (16.67) and the highest R2 (0.38) and EVS (0.47). The regression curves for stable eGFR are displayed in Figure 5A. When classifying stable eGFR into dichotomies by the cutoff value of $45 \mathrm{ml} / \mathrm{min} / 1.73 \mathrm{~m}^{2}$, 

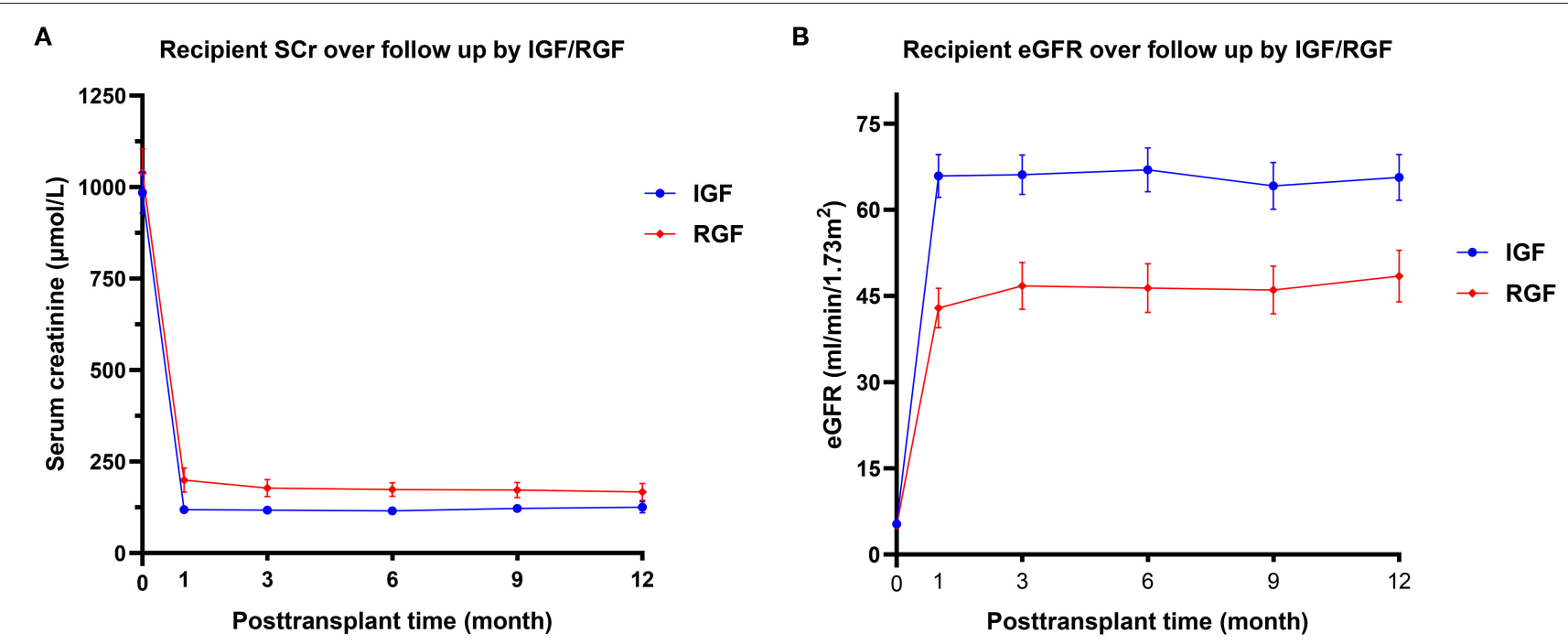

FIGURE 4 | Recipient serum creatinine (A) and eGFR (B) over the first year follow up by immediate graft function (IGF)/RGF. The values of $p$ between IGF and RGF groups at each time point were adjusted by the Holm-Sidak method. Differences in pretransplant serum creatinine and eGFR are not significant, but are significantly different at each time point (adjusted $p<0.01$ ).

TABLE 2 | Prediction of postoperative stable graft function results.

\begin{tabular}{|c|c|c|c|c|c|c|c|c|c|c|c|c|}
\hline \multirow[b]{2}{*}{ Target } & \multirow[b]{2}{*}{ Models } & \multicolumn{4}{|c|}{ Regression index } & \multicolumn{7}{|c|}{ Classification index } \\
\hline & & MAE & RMSE & $\mathbf{R}^{2}$ & EVS & AUC & ACC & PPV & NPV & Sens & Spec & F1 \\
\hline \multirow[t]{6}{*}{ eGFR } & EfficientNet-B5 + CIE & 12.52 & 16.67 & 0.38 & 0.47 & 0.83 & 0.86 & 0.78 & 0.94 & 0.96 & 0.70 & 0.83 \\
\hline & EfficientNet-B5 (image) & 14.68 & 19.32 & 0.31 & 0.41 & 0.73 & 0.74 & 0.71 & 0.78 & 0.85 & 0.61 & 0.73 \\
\hline & $\mathrm{ClE}+\mathrm{PRS}$ & 14.97 & 20.55 & 0.27 & 0.36 & 0.71 & 0.71 & 0.69 & 0.74 & 0.82 & 0.59 & 0.71 \\
\hline & $\mathrm{ClE}$ & 15.07 & 21.37 & 0.25 & 0.35 & 0.69 & 0.70 & 0.68 & 0.72 & 0.81 & 0.57 & 0.69 \\
\hline & Inception-V3 (image) & 15.92 & 22.58 & 0.21 & 0.29 & 0.71 & 0.72 & 0.71 & 0.83 & 0.85 & 0.62 & 0.71 \\
\hline & VGG19 (image) & 18.42 & 28.18 & 0.12 & 0.16 & 0.64 & 0.65 & 0.61 & 0.73 & 0.79 & 0.53 & 0.65 \\
\hline \multirow[t]{6}{*}{ RGF } & EfficientNet-B5 + CIE & & & & & 0.80 & 0.76 & 0.74 & 0.77 & 0.66 & 0.83 & 0.75 \\
\hline & EfficientNet-B5 (image) & & & & & 0.71 & 0.74 & 0.77 & 0.72 & 0.61 & 0.81 & 0.70 \\
\hline & $\mathrm{ClE}+\mathrm{PRS}$ & & & & & 0.70 & 0.68 & 0.58 & 0.74 & 0.56 & 0.76 & 0.68 \\
\hline & $\mathrm{ClE}$ & & & & & 0.66 & 0.66 & 0.62 & 0.68 & 0.55 & 0.77 & 0.64 \\
\hline & Inception-V3 (image) & & & & & 0.65 & 0.73 & 0.61 & 0.68 & 0.56 & 0.76 & 0.65 \\
\hline & VGG19 (image) & & & & & 0.59 & 0.61 & 0.56 & 0.66 & 0.45 & 0.75 & 0.57 \\
\hline
\end{tabular}

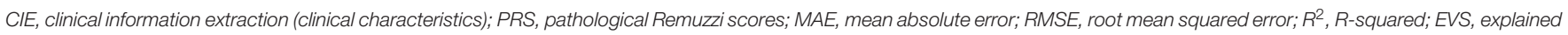
variance score; AUC, area under the ROC curve; ACC, accuracy; PPV, positive predictive value; NPV, negative predictive value; Sens, sensitivity; Spec, specificity; F1, F1 score.

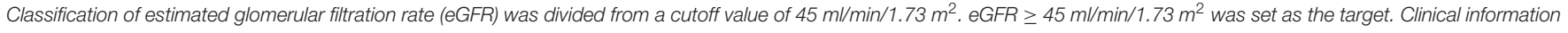
extraction (CIE) included listed clinical features, including donor characteristics, match and preservation features, and recipient characteristics, which are given in Table 1.

the EfficientNet-B5+CIE model significantly improved the predictive performance from an AUC of 0.69 by the CIE model, 0.71 by the CIE+PRS model, and 0.73 by the EfficientNet-B5 model to an AUC of 0.83 by the EfficientNetB5+CIE model (as shown in Figure 5B, Table 2). The EfficientNet-B5+CIE model showed good predictive ability, with a sensitivity of 0.96 , a specificity of 0.70 , a positive predictive value of 0.78 , and a negative predictive value of 0.94 .

For RGF prediction, the EfficientNet-B5+CIE model had the best predictive performance. The pathological image plus clinical characteristics model significantly improved discrimination from an AUC of 0.66 by the CIE model and 0.71 by the EfficientNet-B5 model to an AUC of 0.80 by the EfficientNet-B5+CIE model (Figure 6, Table 2).

\section{Visualization}

To determine from which areas the model extracts the most information, we used gradient-weighted class activation mappings (Grad-CAMs) to explain the results and display them visually. Grad-CAM can translate the output class 


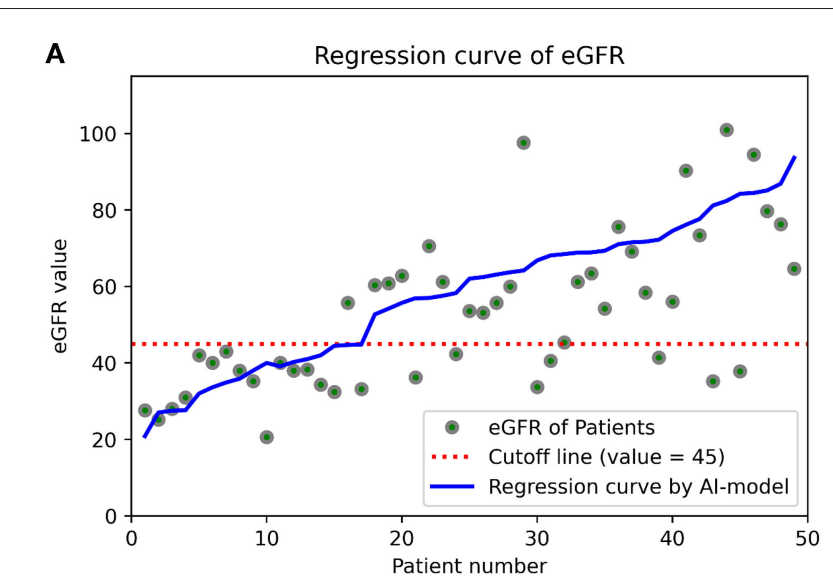

B

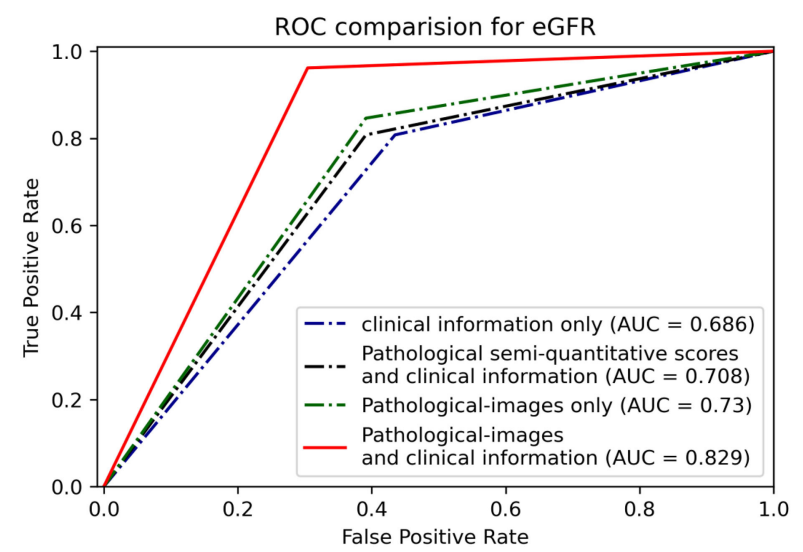

FIGURE 5 | (A) Estimated glomerular filtration rate regression curves. The predictive values of 49 patients are arranged in ascending order, and the corresponding actual values (green dots) and their regression curves (blue lines) are indicated. Cutoff lines (red dashed lines) are also added to facilitate classification. (B) Receiver operating characteristic curves (ROCs) and areas under the ROC curve (AUCs) were used to show the binary classification results of the different models to predict whether eGFR is higher than $45 \mathrm{ml} / \mathrm{min} / 1.73 \mathrm{~m}^{2}$.

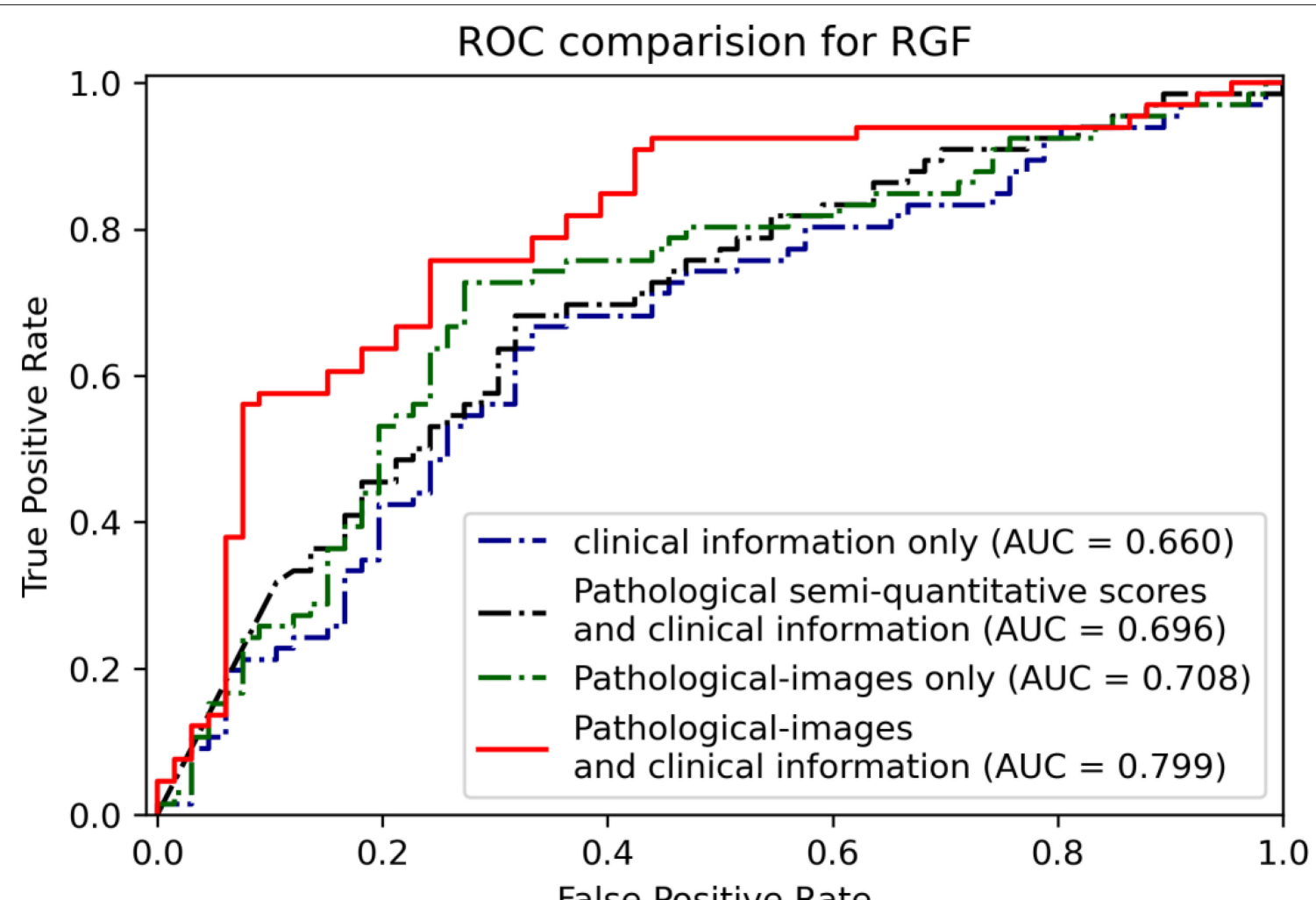

FIGURE 6 | Receiver operating characteristic curves and AUCs show the binary classification results of different models for predicting RGF.

into a final convolutional layer to produce a low-resolution map for a particular category (e.g., RGF) and highlight the discriminative image regions used by models to identify that category. With the help of Grad-CAM, we can judge whether the classification basis of the model is consistent with the clinical scenarios. Some examples of Grad-CAMs are illustrated in Figure 7. The models localized the areas of the tubules and interstitium near the glomeruli, which were discriminative features for classifying RGF from patches. 


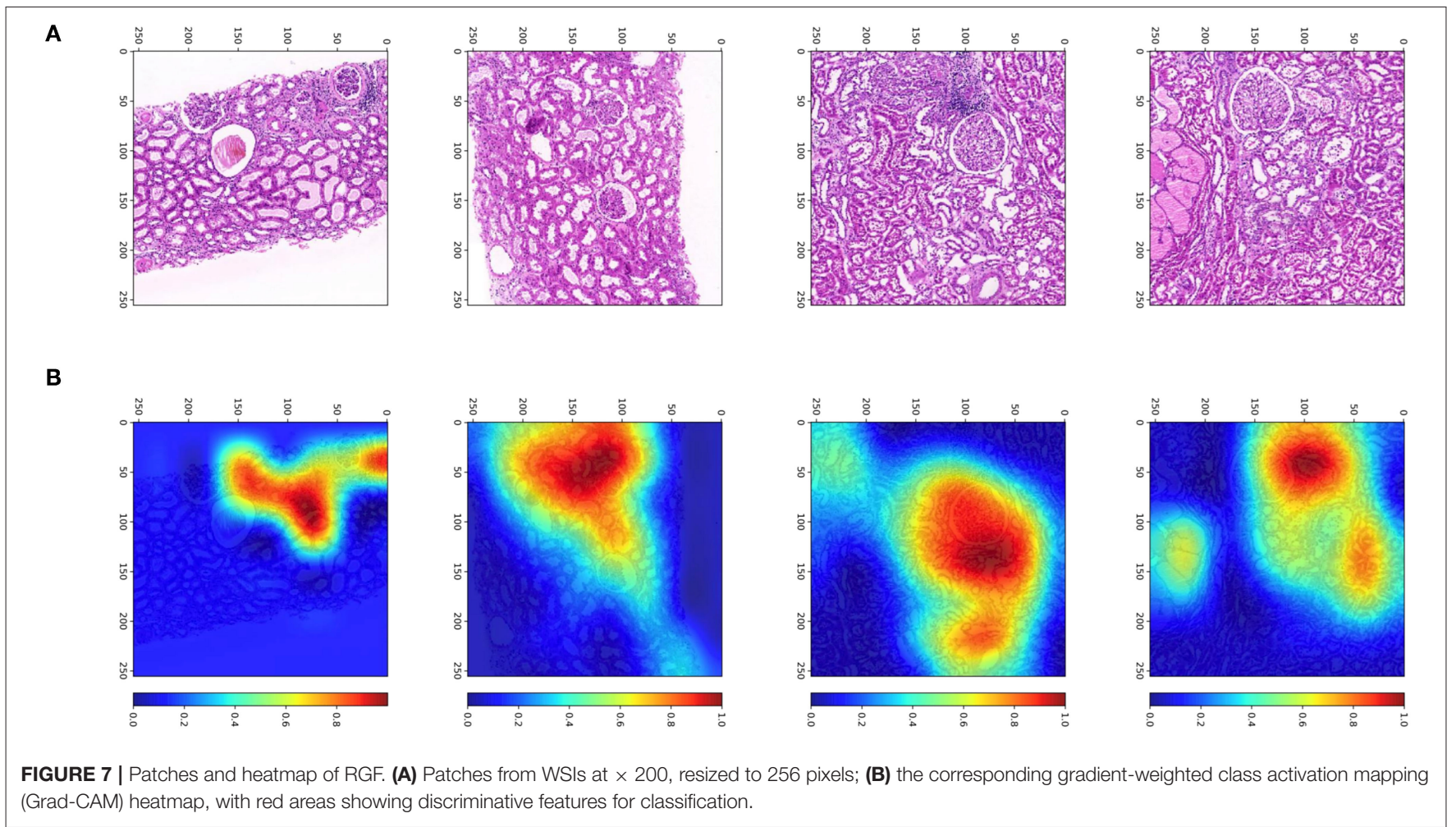

\section{DISCUSSION}

Artificial intelligence (AI) is being widely used in medical scenarios, and with tissue structure inception, there have been some advances in AI studies on renal microstructure histopathology in the last couple of years. For instance, the nephrology and computer science communities have been working on AI for kidney lesion segmentation (1621). Jayapandian et al. (17) indicated that periodic acidSchiff (PAS)-stained WSIs yielded the best concordance in deep learning segmentation. Hermsen et al. (18) presented a CNN for multiclass segmentation of PAS-stained kidney samples, with which the glomeruli, tubules, and interstitium were well-classified. Furthermore, Bouteldja et al. (19) used a deep learning algorithm for multiclass segmentation of PASstained kidney tissue WSIs; the trained CNN segmented six major kidney structures, including the glomerular tuft and glomerulus (such as, Bowman's capsule, tubules, arteries, arterial lumina, and veins), in various species and disease models. Additionally, Uchino et al. (20) developed AI models for seven major pathological lesions, global sclerosis, segmental sclerosis, endocapillary proliferation, mesangial matrix accumulation, mesangial cell proliferation, crescent, and basement membrane structural changes, with the AI model for global sclerosis showing excellent performance. Compared with experienced pathologists, Ligabue et al. (21) achieved a fast speed and comparable accuracy for kidney immunofluorescence reporting by a CNN. The aforementioned deep learning algorithms for glomerulus segmentation were based on PAS-stained or immunohistochemically stained slides. However, properly frozen biopsy slides are preferred in transplantation clinical practice. The CNN-based model developed by Marsh et al. (22) achieved good performance for glomerular sclerosis classification of frozen wedge biopsies. These results indicate the potential application of such models in donor kidney quality evaluation. Nevertheless, there is no study to date on machine learning for the relationship between donor biopsy images and transplant outcomes. Indeed, our study is the first to explore the power of AI on biopsy images and graft prognosis.

For this study, we used donor kidney biopsy WSIs as a feature in addition to clinical characteristics for graft function prediction. Compared with the net clinical characteristics model, the hybrid model displayed the lowest RMSE and MAE. When dividing eGFR by a cutoff of $45 \mathrm{ml} / \mathrm{min} / 1.73 \mathrm{~m}^{2}$, the results showed distinct improvement in the prediction performance of the deep learning algorithms in addition to the clinical characteristics model for independent internal test data. Additionally, the addition of biopsy WSIs improved the prediction accuracy of RGF, and the AUC increased from 0.66 to 0.80 . Overall, donor kidney biopsy WSIs are a useful predictor for graft function recovery. As graft and recipient long-term survival is impacted by posttransplant monitoring and treatment, graft function, complications, dnDSA, chronic rejection, it is difficult to predict long-term survival based on pretransplant donor and recipient parameters. Montero et al. (6) summarized previous prediction models for graft survival, reporting low prediction power in models based on pretransplant features. In general, pretransplant donor scores, such as the deceased donor score (DDS), donor 
risk score (DRS), SCD/ECD, and KDRI/KDPI, have limited predictive performance for graft survival. The discriminative ability (C-index) is approximately $0.6(23,24)$. However, adding posttransplant factors, such as eGFR, proteinuria, acute rejection, and allograft histological parameters, to prediction models significantly increases prediction accuracy (6), which indicates the importance of posttransplant management. Although posttransplant-based models have achieved excellent predictive performance, they are not suitable for pretransplant assessment and decision making. Among posttransplant factors, eGFR plays a critical role in graft survival and recipient quality of life. Functional or well-regenerated kidneys free recipients from dialysis, and a well-regernerated kidney congenitally determines the subsequent incidence of complications as well as the life span of the graft. Many studies have proven that well-regenerated renal function decreases the risk of long-term graft loss $(2,3,15)$. Thus, renal function is deemed as a surrogate endpoint. In this study, we treated RGF and graft function as outcomes of early graft recovery. However, the time point that is suitable for eGFR assessment varies in previous studies $(2,3,15)$. During the first $3 \sim 6$ months, eGFR is not stable because of the need for antibiotics for infection prophylaxis, high-dose immunosuppression agents, and intensive rejection or infection occurrence. After 6 months, these events decrease, and eGFR gradually stabilizes. Most studies have used 1-year renal function as an indicator $(2,15)$ and even eGFR at 3 months $(3)$. In this study, we employed a median value of a best period from each monitored eGFR during $3 \sim 12$ months instead of eGFR at a certain time point. This was independently evaluated, and any obtrusive values with definite causes, such as rejection or infection were excluded.

Among previous DGF prediction models, Irish's DGF nomogram is widely accepted, but the dimensions of the model are complex; its predictive performance is moderate, with a C-index of 0.704 (25). Using bivariate cartography of DGF as an early graft function indicator is arbitrary. In addition, the decreased velocity of serum creatinine plays a critical role in non-DGF recipients. Non-DGF can be divided into SGF and IGF, and among numerous definitions for SGF and IGF, that using serum creatinine at postoperative day 7 (POD7) has the strongest correlation with 12-month graft eGFR (13). Thus, we adopted this definition in this study. Because the incidence of DGF was low and the sample size was small, machine learning with such an imbalanced dataset was hindered. Therefore, we merged the DGF and SGF groups into an RGF group as a surrogate endpoint, as based on a previous study (26). Our results showed a significant improvement in the predictive performance of RGF using the independent internal validation set. This result should be interpreted with caution because our sample size was relatively small, and we did not apply external validation. A multicenter cohort with a large sample size is needed for confirmation. Additionally, we performed heatmap analysis called gradient-weighted class activation mappings (Grad-CAMs) to explore on which feature the machine learning is focused. The results showed that Grad-CAMs focused on the tubules and interstitium near the glomeruli, which were discriminative features for RGF.
For graft function prediction, there is currently no stable predictive algorithm for graft eGFR after transplantation in deceased-donor recipients. The Nyberg score (DDS) is a $0 \sim 39$ scoring system based on feature correlation with 6-month serum creatinine clearance (27). DDS includes donor and HLA matching characteristics, such as donor age, terminal creatinine clearance (by the Cockcroft-Gault equation from age, sex, weight, and terminal serum creatinine), donor hypertension, cause of death, and HLA mismatch for donor quality assessment. Rhu et al. (28) developed a linear model for the prediction of recipient creatinine levels in living-donor transplantation. This linear model incorporates characteristics, such as donor age, donor height, donor serum creatinine, graft weight, recipient sex, recipient height, and recipient weight, showing an $\mathrm{R}^{2}$ of 0.708 , an RMSE of 0.161 , and an intraclass correlation coefficient (ICC) of 0.83 . In addition, the model achieved good prediction performance in the external validation cohort. In another study, Lasserre et al. (7) utilized machine learning approaches to predict transplant graft function outcomes (eGFR after 1 year) via classic clinical characteristics. The Gaussian support vector machine with recursive feature elimination is best for predictions, with a correlation coefficient of 0.48 . When defining transplantation failure with eGFR $<45 \mathrm{ml} / \mathrm{min}$, the AUC was 0.726 . Our model using simple clinical features had a similar AUC of 0.69. Sexton and colleagues tested the discrimination of KDRI/KDPI for eGFR in the Ireland population, and according to the results, KDRI/KDPI was significantly associated with eGFR over 5 years but only accounted for $21 \%$ of eGFR variability over time (24). Overall, the predictive performance of using net clinical features is relatively poor. In this study, a distinct improvement in eGFR classification performance was obtained by using deep learning algorithms for pretransplant biopsy images combined with the clinical characteristics model, with the AUC increasing from 0.69 to 0.83 . When using biopsy images alone, the AUC of deep learning reached 0.73 and surpassed that of the clinical feature model. Although exact prediction (perfect fitting) of graft eGFR is difficult to achieve for deceased-donor recipients, good classification of eGFR can be accurate.

Some limitations exist in this study. First, we used FFPE H\&E-stained WSIs, which differ from frozen H\&E-stained biopsy in terms of slide quality and staining effect. Traditional FFPE H\&E staining preserves morphology with few artifacts but also requires a long processing time. In our center, at least $12 \mathrm{~h}$ were needed to complete the whole FFPE H\&Estained slide preparation, and it is not suitable for the emergent evaluation of donor kidneys due to time constraints. Fast paraffin blocks can be prepared within several hours and is an alternative. Second, this was a retrospective study, and selection bias was inevitable. Pretransplantation biopsy usually occurs in expanded donation or procedural biopsy. Third, ground-truth label imbalance had a negative impact on the predictive model. The number of recipients with eGFR $<45 \mathrm{ml} / \mathrm{min} / 1.73 \mathrm{~m}^{2}$ was much lower than the number for the eGFR $\geq 45 \mathrm{ml} / \mathrm{min} / 1.73 \mathrm{~m}^{2}$ group. Thus, the model favored the prediction of eGFR $\geq 45 \mathrm{ml} / \mathrm{min} / 1.73 \mathrm{~m}^{2}$. Additionally, we labeled only the glomeruli and did not clarify the microstructures of the WSIs in terms of the glomeruli, 
tubules, and interstitium, which would probably provide more detailed information and improve prediction accuracy. Due to the short follow-up times, we did not perform a long-term survival analysis.

Despite the aforementioned limitations, our results preliminarily show that the addition of deep learning for FFPE H\&E-stained WSIs improves the accuracy of graft function prediction in deceased-donor kidney transplant recipients. However, a multicenter prospective study based on frozen biopsy or fast paraffin H\&E-stained WSIs incorporating detailed microstructure recognition is needed for validation.

\section{DATA AVAILABILITY STATEMENT}

The original contributions presented in the study are included in the article/supplementary material, further inquiries can be directed to the corresponding author/s.

\section{ETHICS STATEMENT}

The studies involving human participants were reviewed and approved by Institute Review Board of the Third Affiliated Hospital of Sun Yat-sen University. The Ethics

\section{REFERENCES}

1. Sharma N, Mahajan A, Qazi YA. Marginal kidney transplantation: the road less traveled. Curr Opin Organ Transplant. (2019) 24:926. doi: 10.1097/MOT.0000000000000603

2. Schnitzler MA, Lentine KL, Axelrod D, Gheorghian A, You M, Kalsekar A, et al. Use of 12-month renal function and baseline clinical factors to predict long-term graft survival: application to BENEFIT and BENEFIT-EXT trials. Transplantation. (2012) 93:172-81. doi: 10.1097/TP.0b013e31823ec02a

3. Mottola C, Girerd N, Duarte K, Aarnink A, Giral M, Dantal J, et al. Prognostic value for long-term graft survival of estimated glomerular filtration rate and proteinuria quantified at 3 months after kidney transplantation. Clin Kidney J. (2020) 13:791-802. doi: 10.1093/ckj/sfaa044

4. Baek CH, Kim H, Yang WS, Han DJ, Park SK. A postoperative 1-Year eGFR of more than $45 \mathrm{ml} / \mathrm{min}$ may be the cutoff level for a favorable long-term prognosis in renal transplant patients. Ann Transplant. (2016) 21:439-47. doi: 10.12659/AOT.897938

5. Marcen R, Dal Canton A. Glomerular filtration rate: utility for assessing longterm renal allograft outcomes in kidney allograft recipients. J Nephrol. (2013) 26:1009-24. doi: 10.5301/jn.5000308

6. Montero N, Codina S, Cruzado JM. Prediction scores for risk of allograft loss in patients receiving kidney transplants: nil satis nisi optimum. Clin Kidney J. (2020) 13:745-8. doi: 10.1093/ckj/sfaa081

7. Lasserre J, Arnold S, Vingron M, Reinke P, Hinrichs C. Predicting the outcome of renal transplantation. $J \mathrm{Am}$ Med Inform Assoc. (2012) 19:255-62. doi: 10.1136/amiajnl-2010-0 00004

8. Salvadori M, Tsalouchos A. Histological and clinical evaluation of marginal donor kidneys before transplantation: which is best? World J Transplant. (2019) 9:62-80. doi: 10.5500/wjt.v9.i4.62

9. Moeckli B, Sun P, Lazeyras F, Morel P, Moll S, Pascual M, et al. Evaluation of donor kidneys prior to transplantation: an update of current and emerging methods. Transpl Int. (2019) 32:459-69. doi: 10.1111/tri.13430

10. Liapis H, Gaut JP, Klein C, Bagnasco S, Kraus E, Farris AB, et al. Banff histopathological consensus criteria for preimplantation kidney biopsies. Am J Transplant. (2017) 17:140-50. doi: 10.1111/ajt.13929
Committee waived the requirement of written informed consent for participation.

\section{AUTHOR CONTRIBUTIONS}

$\mathrm{NN}$ and YR conceived of and designed this study. YL, JL, ZT, $\mathrm{JZ}, \mathrm{ZD}, \mathrm{WD}$, and BM collected the data and biopsy slides. LH, $\mathrm{YR}$, and YL analyzed the data. YL and XH wrote the manuscript. $\mathrm{BM}$ and NN supervised and revised the manuscript. All authors contributed to the article and approved the submitted version.

\section{FUNDING}

This work was supported by the National Natural Science Foundation of China (81970652 and 81702409), the Guangdong Basic and Applied Basic Research Foundation (2019A1515011219), the Bioengineering Research Center Training Project of the Third Affiliated Hospital of Sun Yatsen University (SW201904), the Third Affiliated Hospital of Sun Yat-sen University Clinical Research Program (YHJH201906), the Science and Technology Planning Project of Guangzhou (201803010016), and the Guangdong Provincial Key Laboratory of Digestive Cancer Research (No. 2021B1212040006).
11. Niel O, Bastard P. Artificial intelligence in nephrology: core concepts, clinical applications, and perspectives. Am J Kidney Dis. (2019) 74:80310. doi: $10.1053 / j . a j k d .2019 .05 .020$

12. Mallon DH, Summers DM, Bradley JA, Pettigrew GJ. Defining delayed graft function after renal transplantation: simplest is best. Transplantation. (2013) 96:885-9. doi: 10.1097/TP.0b013e3182a19348

13. Hall IE, Reese PP, Doshi MD, Weng FL, Schroppel B, Asch WS, et al. Delayed graft function phenotypes and 12-month kidney transplant outcomes. Transplantation. (2017) 101:1913-23. doi: 10.1097/TP.0000000000001409

14. Levey AS, Stevens LA, Schmid CH, Zhang YL, Castro AF, 3rd, Feldman HI, et al. A new equation to estimate glomerular filtration rate. Ann Intern Med. (2009) 150:604-12. doi: 10.7326/0003-4819-150-9-200905050-00006

15. Kasiske BL, Israni AK, Snyder JJ, Skeans MA, Patient Outcomes in Renal Transplantation I. The relationship between kidney function and long-term graft survival after kidney transplant. Am J Kidney Dis. (2011) 57:46675. doi: 10.1053/j.ajkd.2010.10.054

16. Barisoni L, Lafata KJ, Hewitt SM, Madabhushi A, Balis UGJ. Digital pathology and computational image analysis in nephropathology. Nat Rev Nephrol. (2020) 16:669-85. doi: 10.1038/s41581-020-0321-6

17. Jayapandian CP, Chen Y, Janowczyk AR, Palmer MB, Cassol CA, Sekulic $\mathrm{M}$, et al. Development and evaluation of deep learning-based segmentation of histologic structures in the kidney cortex with multiple histologic stains. Kidney Int. (2021) 99:86-101. doi: 10.1016/j.kint.2020.07.044

18. Hermsen M, de Bel T, den Boer M, Steenbergen EJ, Kers J, Florquin S, et al. Deep learning-based histopathologic assessment of kidney tissue. J Am Soc Nephrol. (2019) 30:1968-79. doi: 10.1681/ASN.2019020144

19. Bouteldja N, Klinkhammer BM, Bulow RD, Droste P, Otten SW, Freifrau von Stillfried S, et al. Deep learning-based segmentation and quantification in experimental kidney histopathology. J Am Soc Nephrol. (2021) 32:5268. doi: 10.1681/ASN.2020050597

20. Uchino E, Suzuki K, Sato N, Kojima R, Tamada Y, Hiragi S, et al. Classification of glomerular pathological findings using deep learning and nephrologist-AI collective intelligence approach. Int J Med Inform. (2020) 141:104231. doi: 10.1016/j.ijmedinf.2020.104231

21. Ligabue G, Pollastri F, Fontana F, Leonelli M, Furci L, Giovanella S, et al. Evaluation of the classification accuracy of the kidney biopsy direct 
immunofluorescence through convolutional neural networks. Clin J Am Soc Nephrol. (2020) 15:1445-54. doi: 10.2215/CJN.03210320

22. Marsh JN, Matlock MK, Kudose S, Liu TC, Stappenbeck TS, Gaut JP, et al. Deep learning global glomerulosclerosis in transplant kidney frozen sections. IEEE Trans Med Imaging. (2018) 37:2718-28. doi: 10.1109/TMI.2018.2851150

23. Peters-Sengers H, Heemskerk MBA, Geskus RB, Kers J, Homan van der Heide $\mathrm{JJ}$, Berger SP, et al. Validation of the prognostic kidney donor risk index scoring system of deceased donors for renal transplantation in the Netherlands. Transplantation. (2018) 102:162-70. doi: 10.1097/TP.0000000000001889

24. Sexton DJ, O'Kelly P, Kennedy C, Denton M, de Freitas DG, Magee C, et al. Assessing the discrimination of the Kidney Donor Risk Index/Kidney Donor Profile Index scores for allograft failure and estimated glomerular filtration rate in Ireland's National Kidney Transplant Programme. Clin Kidney J. (2019) 12:569-73. doi: $10.1093 / \mathrm{ckj} / \mathrm{sfy} 130$

25. Irish WD, Ilsley JN, Schnitzler MA, Feng S, Brennan DC. A risk prediction model for delayed graft function in the current era of deceased donor renal transplantation. Am J Transplant. (2010) 10:227986. doi: 10.1111/j.1600-6143.2010.03179.x

26. Johnston O, O'Kelly P, Spencer S, Donohoe J, Walshe JJ, Little $\mathrm{DM}$, et al. Reduced graft function (with or without dialysis) vs immediate graft function-a comparison of long-term renal allograft survival. Nephrol Dial Transplant. (2006) 21:2270-4. doi: 10.1093/ndt/g fl103

27. Nyberg SL, Matas AJ, Kremers WK, Thostenson JD, Larson TS, Prieto $\mathrm{M}$, et al. Improved scoring system to assess adult donors for cadaver renal transplantation. Am J Transplant. (2003) 3:715-21. doi: 10.1034/j.1600-6143.2003.00111.x

28. Rhu J, Kim SJ, Lee KW, Park JB, Kim K, Yoo H, et al. Development of a novel linear model for predicting recipient's post-transplant serum creatinine level after living donor kidney transplantation: a multicenter cross-validation study. PLoS ONE. (2019) 14:e0214247. doi: 10.1371/journal.pone.0214247

Conflict of Interest: The authors declare that the research was conducted in the absence of any commercial or financial relationships that could be construed as a potential conflict of interest.

Publisher's Note: All claims expressed in this article are solely those of the authors and do not necessarily represent those of their affiliated organizations, or those of the publisher, the editors and the reviewers. Any product that may be evaluated in this article, or claim that may be made by its manufacturer, is not guaranteed or endorsed by the publisher.

Copyright $\odot 2022$ Luo, Liang, Hu, Tang, Zhang, Han, Dong, Deng, Miao, Ren and $\mathrm{Na}$. This is an open-access article distributed under the terms of the Creative Commons Attribution License (CC BY). The use, distribution or reproduction in other forums is permitted, provided the original author(s) and the copyright owner(s) are credited and that the original publication in this journal is cited, in accordance with accepted academic practice. No use, distribution or reproduction is permitted which does not comply with these terms. 